\title{
Evaluation of the presence of SARS-CoV-2 in the vaginal fluid of reproductive-aged women
}

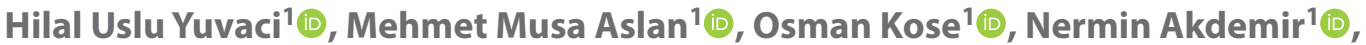

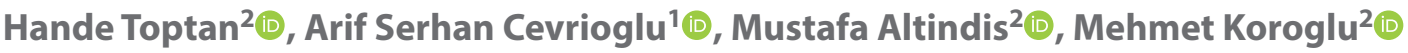 \\ ${ }^{1}$ Department of Obstetrics and Gynecology, Sakarya Training and Research Hospital, , Sakarya, Turkey \\ ${ }^{2}$ Department of Microbiology, Sakarya Training and Research Hospital, Sakarya, Turkey
}

\begin{abstract}
Objectives: Severe Acute Respiratory Syndrome Coronavirus 2 (SARS-CoV-2) is mainly transmitted through respiration and direct contact. The status of the infection in the female genital system is still unknown. The study aimed to evaluate whether SARS-CoV-2 is present in the vaginal fluid of women with COVID-19 infection in reproductive period.

Material and methods: Women who were between the ages of 18-50 years and clinically confirmed to have COVID-19 infection at our hospital between 20 April-31 May 2020 were included in the study. Women who were in their menstrual cycle during the study and who had a known cervical intraepithelial lesion and/or cancer, sexually transmitted disease and history and/or symptoms of vaginitis were excluded from the study. In patients in whom no pathology was detected during the examination, a sample was taken from the vaginal fluid for PCR by using Dacron tip swab. Analysis was performed with Genesig Real-Time PCR COVID-19 kit (Primer Design, England).

Results: Eighteen women who were in reproductive period and diagnosed with severe COVID-19 pneumonia were included in the study. The mean age of the patients was $38.16 \pm 8.54$. None of the patients were in their menopause period. The clinical symptoms of these women were similar to those of confirmed severe COVID-19 cases. SARS-CoV- 2 was found to be negative in the samples taken from the vaginal fluid in all patients.

Conclusions: SARS-CoV-2 virus was not detected in the vaginal fluid of the patients who tested positive for COVID-19 in reproductive period.
\end{abstract}

Key words: coronavirus; SARS-CoV-2; vaginal fluid

Ginekologia Polska 2021; 92, 6: 406-409

\section{INTRODUCTION}

Viral pneumonia and severe acute respiratory symptom observed in coronavirus disease (COVID-19) have spread between many countries in a short period of time and has affected the whole world, causing a pandemic to be declared. Severe Acute Respiratory Syndrome Coronavirus 2 (SARS-CoV-2), which is the pathogen of the coronavirus disease, is a highly contagious virus, and it is mainly transmitted through the respiratory droplets of people in the immediate vicinity and through direct contact [1]. The virus especially affects the upper and lower respiratory systems in human body. It can lead to fever, cough, chest pain, dyspnea, fatigue, and muscle pains [2]. In addition, it has also been known to cause abdominal pain and diarrhea by affecting the gastrointestinal system, which can lead to acute cardiac destruction, arrhythmia and shock symptoms in the cardiovascular system, as well as mental fog and confusion in the central nervous system, and patients facing mortality resulting from multiple organ failure [3]. However, it is still not clearly determined whether it creates infection in the female genital system. There are few studies conducted in the literature on the presence of SARS-CoV-2 virus in body fluids. It has been observed that very few women in post-menopausal period were included in the studies investigating the virus in the vaginal fluid [4].

\section{Objectives}

Determining this virus in the genital systems of women during the reproductive period is important in that it may help reporting especially the risk of transmission through sexual intercourse and from mother to baby during delivery. The purpose of the study was to investigate whether

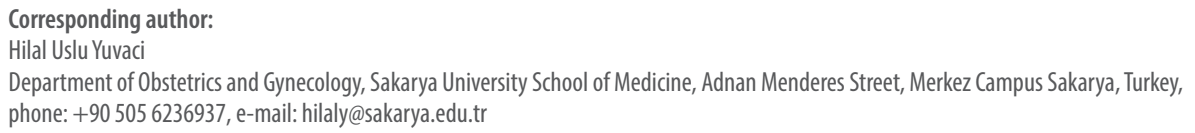


there are SARS-CoV-2 virus symptoms in the vaginal fluids of women who are sexually active and in the reproductive period.

\section{MATERIAL AND METHODS}

Study group and ethical approval

In this cross-sectional prospective study, sexually active menstruating women between the ages of 18-50 years who were hospitalized in the COVID-19 services of Sakarya University Training and Research Hospital, which was transformed into a pandemic hospital where COVID-19 patients were treated and followed up, in Sakarya Province of Turkey. Patients who were clinically confirmed to have COVID-19 infection (whose swap sample taken from the throat tested positive in PCR test) between 20 April-31 May 2020 were included in the study. Patients who were in their menstrual cycle during the study, who had a diagnosed cervical intraepithelial lesion and/or cancer, sexually transmitted disease, and vaginitis history and symptoms were excluded from the study. All patients were informed about the study, and after obtaining their verbal/written approval, they were evaluated in lithotomy position. Ethical approval for the study was taken from Sakarya University Medical Faculty Ethical Committee (Project ID:16214662/050.01.04/83) (April 13, 2020).

\section{Taking sample from the vaginal fluid}

Within 0-2 days of hospitalization, samples were taken from the vaginal fluids for PCR test by using Dacron (Polyester) tip swab from patients in whom no pathology was observed in the examination made after insertion of vaginal speculum in lithotomy position on the examination table. The swab sample was taken from the vaginal fluid by applying the swab on the anterior, posterior and lateral walls once, and touching the posterior fornix once. All samples taken were placed into screw-capped sealed vessels which contained $3 \mathrm{~mL}$ of viral transport medium. All samples were kept at $2-8^{\circ} \mathrm{C}$ in refrigerator right after taking the samples and were transported quickly to the laboratory. The samples were sent to the laboratory by complying with infection protection and control procedures and cold chain rules in triple transportation system.

\section{Nucleic acid isolation and Reverse Transcriptase Polymerase Chain Reaction (RT-PCR)}

After the samples were accepted at the microbiology laboratory, all samples were studied in biosecurity cabin in level 3 negative pressure room.

After the vaginal fluid samples were well vortexed, $400 \mu \mathrm{L}$ was removed and loaded onto BioRobot EZ1 (Qiagen, Germany) device, and $60 \mu \mathrm{L}$ elution was taken. Total nucleic acid isolation was performed with EZ1 Virus Mini Kit
Table 1. COVID-19 RT-PCR temperature and duration adjustments

\begin{tabular}{|l|l|l|l|}
\hline Stages & Temperature & Duration \\
\hline Reverse transcription & $55^{\circ} \mathrm{C}$ & 10 minutes \\
\hline Enzyme activation & $95^{\circ} \mathrm{C}$ & 2 minutes \\
\hline \multirow{2}{*}{ X50 Cycle } & Denaturation & $95^{\circ} \mathrm{C}$ & 10 seconds \\
\cline { 2 - 4 } & Binding and Stretching & $60^{\circ} \mathrm{C}$ & 60 seconds \\
\hline
\end{tabular}

v2.0 (Qiagen, Germany) in accordance with the company's recommendations.

For RT-PCR study, a total mixture with a volume of $20 \mu \mathrm{L}$ made up of $10 \mu \mathrm{L}$ master mix, $2 \mu \mathrm{L}$ primary, and $8 \mu \mathrm{L}$ RNA template was formed, and reaction was realized with a total of $20 \mu \mathrm{L}$ reaction volume in the durations and at the temperatures given in Table 1.

At the end of the reaction, the curves whose Cycle Threshold (CT) value was lower than 45 and were observed to be sigmoidal were interpreted as positive for SARS-CoV-2 RNA.

\section{RESULTS}

Eighteen women in reproductive period with severe COVID-19 pneumonia were included in the study. The mean age of the patients was $38.16 \pm 8.54$. None of the patients were in their menstrual cycle. The clinical symptoms of these patients were comparable to those of confirmed COVID-19 patients. The most common symptoms were fever $(100 \% \mathrm{n}=18)$ and cough $(66.6 \% \mathrm{n}=12)$. Other symptoms included sore throat $(44.4 \% \mathrm{n}=8)$, fatigue $(27.7 \% \mathrm{n}=5)$, and diarrhea $(16.7 \% \mathrm{n}=3)$. Two patients complained of dyspnea, and no patients complained about urinary tract symptoms. While no chronic diseases were observed in the patients, hypertension $(n=5)$ and diabetes $(n=1)$ were present in some patients. Computer tomography scans of the patents were observed to be consistent with typical viral pneumonia. The swab samples taken from the throats of the patients at the time of hospitalization tested positive in PCR. PCR tests of these patients repeated after 24 hours also tested positive. ICU treatment was not required for any of the patients. For all patients, treatment with antiviral (oseltamivir/favipiravir) and antibacterial (azithromycin and hydroxychloroquine) drugs were initiated. Baseline characteristics of the 18 patients infected with SARS-CoV-2 are presented in Table 2. The samples taken from the vaginal fluid tested negative for SARS-CoV-2 in all patients.

\section{DISCUSSION}

In our study, viral RNA was not determined in the vaginal fluids of the COVID-19 positive women during the reproductive period with a mean age of $38.16 \pm 8.54$. The virus was 
Table 2. Baseline characteristics of the patients infected with SARS-CoV-2

\begin{tabular}{|l|l|}
\hline Age $($ mean \pm SD) & $38.16 \pm 8.54$ \\
\hline Fever $(n, \%)$ & $18(100 \%)$ \\
\hline Cough $(n, \%)$ & $12(66.6 \%)$ \\
\hline Sore throat $(n, \%)$ & $8(44.4 \%)$ \\
\hline Fatigue $(n, \%)$ & $5(27.7 \%)$ \\
\hline Diarrhea $(n, \%)$ & $3(16.7 \%)$ \\
\hline Hypertension $(n, \%)$ & $5(27.7 \%)$ \\
\hline Diabetes Mellitus $(n, \%)$ & $1(5.5 \%)$ \\
\hline Samples & $1.33(0-2$ days) \\
\hline Discharge & $7.33(5-12$ days) \\
\hline
\end{tabular}

not detected in the vaginal fluid in the studies conducted on this issue in the literature as well. What makes our study different is that the patients included in the study were younger and in the reproductive period. In a similar study in which 12 pregnant women with a mean age of $32 \pm 7.9$ were included, the virus could not be shown in the vagina through isolation methods [5].

In a study carried out by Qui et al. [4], SARS-CoV-2 was investigated with PCR in the vaginal fluid samples of 10 patients aged between 50-80 in their postmenopausal period who tested positive for COVID-19, and no virus was found. Moreover, in a study in which 35 patients, 28 of whom were in the postmenopausal period, were included, SARS-CoV-2 similarly could not be detected in the vaginal fluids [6]. According to International Menopause Society (IMS), it has been reported that within 4-5 years following menopause, urogenital atrophy and vaginal dryness develop in most women [7]. Atrophy and dryness that develop in the vaginal epithelium as a result of hormonal changes in menopause may have caused lack of material, and PCR test results may have proved negative in these studies.

SAR-CoV-2 is an enveloped RNA virus. It has been demonstrated that SARS-CoV-2 enters the body by binding to angiotensin-converting enzyme 2 receptors (ACE2) expressed in vascular endothelial membrane in many organs such as lungs, gastrointestinal system, kidney and testicles by spike proteins, and causes infection [8]. These ACE2 receptors have been shown to be non-existing in the vaginal epithelium [9]. This could be the reason why neither our study nor other studies could detect the virus in the vagina.

The potential for a patient to spread the infection requires the virus to be present in different body fluids. In the literature, the virus has been isolated in the urine, feces, nasopharyngeal swab sample and blood [10]. In a study conducted, it has been shown that although the clinical symptoms of the patents improved and PCR tested negative for the swab sample taken from the throat, the virus was found to be present in the samples taken in later periods and to continue spreading [11]. In our study, the samples were taken in early period and the virus could not be isolated in the vaginal fluid. Accordingly, since the invasion duration of the virus in the tissues is not known, the presence of the virus should be evaluated by taking samples from the vaginal fluid in later periods.

The limitations of our study are that the number of patients included in the study was low, the patients were not evaluated together with their sexual partners, the patients did not have serious diseases that required intensive care though they tested positive in PCR, and the vaginal fluid samples were taken in the first days of hospitalization (0-2 days) at the beginning of the treatment. There is a need for further studies in which vaginal samples taken in later periods from women who were clinically confirmed to be infected with COVID-19 through PCR are compared.

\section{CONCLUSIONS}

In our study, SARS-CoV-2 virus could not be detected in the vaginal fluids of female patients during the reproductive period who tested positive for COVID-19 infection. Our results are important in that they show that the lower genital system in infected women in reproductive period is not a mode of transmission for SAR-CoV-2. In addition, our results can provide a guidance for the choice of delivery method in infected pregnant women in the management of SARS-CoV-2. Randomized controlled studies with large samples are needed regarding this issue.

\section{Conflict of interest}

The authors declare no conflict of interest.

\section{REFERENCES}

1. Chan JW, Yuan S, Kok KH, et al. A familial cluster of pneumonia associated with the 2019 novel coronavirus indicating person-to-person transmission: a study of a family cluster. The Lancet. 2020; 395(10223): 514-523, doi: 10.1016/s0140-6736(20)30154-9.

2. Zhu Na, Zhang D, Wang W, et al. China Novel Coronavirus Investigating and Research Team. A Novel Coronavirus from Patients with Pneumonia in China, 2019. N Engl J Med. 2020; 382(8): 727-733, doi: 10.1056/NEJMoa2001017, indexed in Pubmed: 31978945.

3. Harapan H, Itoh N, Yufika A, et al. Coronavirus disease 2019 (COVID-19): A literature review. J Infect Public Health. 2020; 13(5): 667-673, doi: 10.1016/j.jiph.2020.03.019, indexed in Pubmed: 32340833.

4. Qiu L, Liu X, Xiao M, et al. SARS-CoV-2 Is Not Detectable in the Vaginal Fluid of Women With Severe COVID-19 Infection. Clin Infect Dis. 2020; 71(15): 813-817, doi: 10.1093/cid/ciaa375, indexed in Pubmed: 32241022.

5. Aslan MM, Uslu Yuvacı H, Köse O, et al. SARS-CoV-2 is not present in the vaginal fluid of pregnant women with COVID-19. J Matern Fetal Neonatal Med. 2020 [Epub ahead of print]: 1-3, doi: 10.1080/14767058.2020.1793318, indexed in Pubmed: 32669014 
6. Cui $P, C$ Chen $Z$, Wang T, et al. Severe acute respiratory syndrome coronavirus 2 detection in the female lower genital tract. Am J Obstet Gynecol. 2020;223(1): 131-134, doi: 10.1016/j.ajog.2020.04.038, indexed in Pubmed: 32376320.

7. Sturdee DW, Panay N. International Menopause Society Writing Group. Recommendations for the management of postmenopausal vaginal atrophy. Climacteric. 2010; 13(6): 509-522, doi: 10.3109/13697137.2010.522875, indexed in Pubmed: 20883118.

8. Riordan JF. Angiotensin-I-converting enzyme and its relatives. Genome Biol. 2003; 4(8): 225, doi: 10.1186/gb-2003-4-8-225, indexed in Pubmed: 12914653.
9. Cui $P$, Chen $Z$, Wang $T$, et al. Clinical features and sexual transmission potential of SARS-CoV-2 infected female patients: a descriptive study in Wuhan, China. , doi: 10.1101/2020.02.26.20028225.

10. Peng L, Liu J, Xu W, et al. SARS-CoV-2 can be detected in urine, blood, anal swabs, and oropharyngeal swabs specimens. J Med Virol. 2020; 92(9): 1676-1680, doi: 10.1002/jmv.25936, indexed in Pubmed: 32330305.

11. Chen $Y, C$ hen $L$, Deng $Q$, et al. The presence of SARS-CoV-2 RNA in the feces of COVID-19 patients. J Med Virol. 2020; 92(7): 833-840, doi: 10.1002/jmv.25825, indexed in Pubmed: 32243607. 